problem of insertional mutagenesis. We know that insertional mutagenesis can induce cancer ${ }^{1,2}$ and may disrupt genes that affect the fitness of the organism unrelated to cancer $^{3}$. Ansari proposes an alternative approach in which cell-penetrating peptides are fused to artificial transcription factors. This method potentially suffers from the above limitations along with the inability to maintain stable transcription factor levels.

An alternative, not mentioned in the article that is currently being investigated by our group and others, is targeting transgenic integration via chimeric integrases ${ }^{4}$ or transposases. The ability to add a highly specific zinc-finger domain or substitute the enzyme's native host DNA binding domain to achieve targeted integration exists and is being explored.

Transposon-based constructs have many advantages over retroviruses. They are DNA, frequently in plasmid form, and packaged nonvirally, thus allowing increased stability. Furthermore, a DNA construct is preferred because the reverse transcriptase of a retrovirus has high error rates in copying both RNA and DNA because it lacks the $3^{\prime} \rightarrow 5^{\prime}$ exonuclease activity necessary for proofreading during DNA synthesis.
Joseph Kaminski and James Bradley Summers, Transpovec Corporation, 800 Cottman Avenue,

Suite 429,

Philadelphia, PA 19111

(JM_Kaminski@fccc.edu)

1. Suzuki, T. et al. Nat. Genet. 32, 166-174 (2002).

2. Marwick, C. Br. Med. J. 326, 181 (2003).

3. Catteruccia, F., Godfray, H.C. \& Crisanti, A. Science 299, 1225-1227 (2003).

4. Peng, W.J., Chang, C.M. \& Lin, T.H. J. Biomed Sci. 9, 171-184 (2002).

\section{GMO opposition not based on a mistake}

To the editor:

In his commentary in the March issue, "Transgenic organisms-time for conceptual diversification?" (Nat. Biotechnol. 21, 227-228, 2003), Nielsen hypothesizes that a system of measuring different degrees of genetic distance "may ultimately lead to increased public acceptance" of genetically modified organisms (GMOs). He thinks this because he is guessing that "misleading conceptual assumptions" are behind the negative perceptions of GMOs. His hypothesis is a shot in the dark, and it shows that Nielsen, like many advocates, has no idea what upsets the critics of GMOs.

The misleading assumption here is his own assumption that " $\mathrm{t}] \mathrm{he}$ extent to which transgenic organisms differ from traditionally bred organisms underlies much of the controversy surrounding the use of GMOs." This is not what underlies the controversy, and people are not compulsive purists, anxiously struggling to keep separate species in separate boxes.

What underlies the controversy is whether crop germplasm is public domain or is privately owned through patents on plants and animals. If scientists really want to address the root of opposition to transgenic food, they first need to acknowledge what that underlying root is: monopoly control of the world's food supply. Finely graded scales and nuanced distinctions like Nielsen describes are sometimes just what we need. But not in this case. In the GMO controversy, the solutions may be difficult, but the key distinction is not. It really is up or down, black or white, as definite as whether a patent office says 'yes' or 'no.'

Jerry Cayford, Resources for the Future, 1616 P Street, NW, Washington, DC 20036 (cayford@rff.org) 